\title{
Visual Quality Improvement of Medical Images using Pixel Reconstruction Followed by Gabor Enhancement Technique
}

\author{
Pooja Gupta and Kuldip Pahwa \\ Department of Electronics and Communication, MMEC MMU, Mullana, Ambala, \\ Haryana, India \\ pooja.kaushik@mmumullana.org,kuldip.pahwa@mmumullana.org
}

\begin{abstract}
Today is an era of digital imaging. This can be viewed either in the field of photography or in the field of medical imaging. Digital imaging has improved the performance of picture quality. Detailed information can be recovered very quickly from any part of an image and this feature has become very useful in every field of imaging. This improvement in the field of medical imaging has given life to so many patients as diagnosis of disease has become very fast and easy. But many a times the image quality is not upto the mark, due to this reason; the doctors are not able to diagnose the disease. So this paper proposes a noval approach for improvement in quality of medical images using pixel reconstruction followed by Gabor filter enhancement technique. The experimental results are verified as improvement in PSNR of hexagonal pixel images as compared with square pixel images. The results show a large improvement in quality of digital imaging.
\end{abstract}

Key words: digital imaging, image enhancement, medical imaging, Gabor filter

\section{Introduction}

Medical imaging is becoming very helpful now a day, because of its numerous applications like MRI, CT scan, X-Ray, ultrasound, ECG, etc. Thus detection of disease has become convenient for radiologists and surgeons, just because of real time applications in medical imaging. High quality imaging equipments and devices are available in the market, which are helpful in problem detection and identification. But sometimes due to poor picture quality it becomes difficult to differentiate or detect a disease. At such situations image processing plays a vital role to enhance the image and improves its quality; and develops a quality output (picture). Literature reveals that a number of image enhancement techniques are applied on medical imaging; but quality of enhanced picture does not show good improvement. This paper deals with the improvement in medical image quality using pixel reconstruction followed by Gabor filter enhancement technique. The rest of the paper is organised in the following way. Section 2 presents the proposed work methodology. Section 3 deals with the results and discussions. Section 4 briefs the conclusion. 


\section{Work methodology}

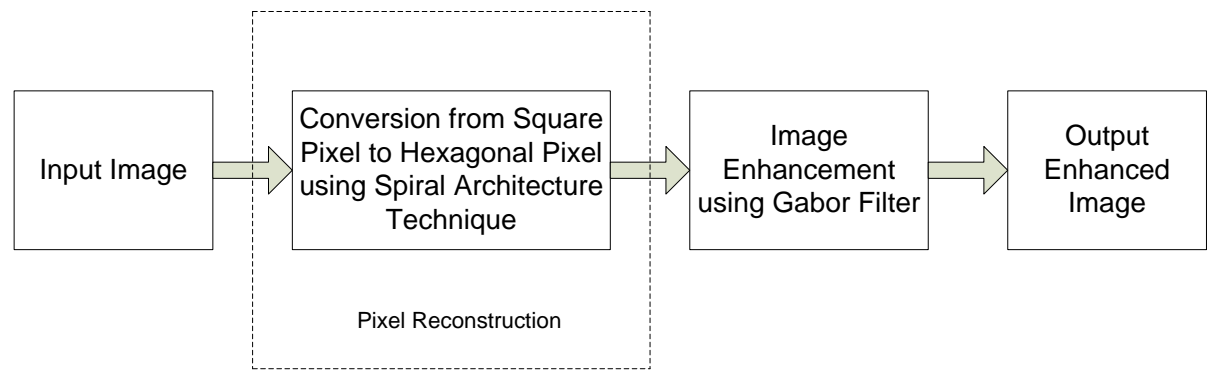

Figure 1. Block diagram of proposed approach

\section{Step 1: Pixel Reconstruction}

Conversion from square pixel to hexagonal pixel using spiral architecture technique [3].

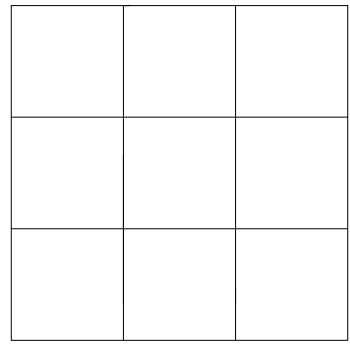

Square Pixel Image

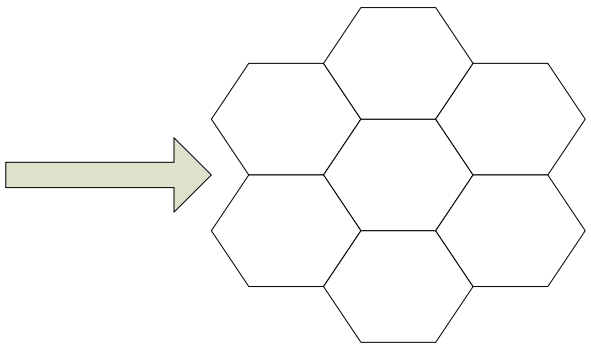

Hexagonal Pixel Image

Figure 2. Pixel reconstruction from square structure to hexagonal structure

The hexagonal pixel is designed by dividing a square pixel into $7 \times 7$ sub-pixels; while maintaining intensity of sub-pixels same as that of the pixel from which the sub-pixels are obtained. Finally a hexagonal pixel is formed using 56 sub- pixels arranged as shown in Figure 3.

\begin{tabular}{|l|l|l|l|l|l|l|l|l|}
\hline & & $\mathrm{X}_{0}$ & $\mathrm{X}_{0}$ & $\mathrm{X}_{0}$ & $\mathrm{X}_{0}$ & $\mathrm{X}_{0}$ & & \\
\hline & $\mathrm{X}_{0}$ & $\mathrm{X}_{0}$ & $\mathrm{X}_{0}$ & $\mathrm{X}_{0}$ & $\mathrm{X}_{0}$ & $\mathrm{X}_{0}$ & $\mathrm{X}_{0}$ & \\
\hline & $\mathrm{X}_{0}$ & $\mathrm{X}_{0}$ & $\mathrm{X}_{0}$ & $\mathrm{X}_{0}$ & $\mathrm{X}_{0}$ & $\mathrm{X}_{0}$ & $\mathrm{X}_{0}$ & \\
\hline $\mathrm{X}_{0}$ & $\mathrm{X}_{0}$ & $\mathrm{X}_{0}$ & $\mathrm{X}_{0}$ & $\mathrm{X}_{0}$ & $\mathrm{X}_{0}$ & $\mathrm{X}_{0}$ & $\mathrm{X}_{0}$ & $\mathrm{X}_{0}$ \\
\hline $\mathrm{X}_{0}$ & $\mathrm{X}_{0}$ & $\mathrm{X}_{0}$ & $\mathrm{X}_{0}$ & $\mathrm{X}_{0}$ & $\mathrm{X}_{0}$ & $\mathrm{X}_{0}$ & $\mathrm{X}_{0}$ & $\mathrm{X}_{0}$ \\
\hline & $\mathrm{X}_{0}$ & $\mathrm{X}_{0}$ & $\mathrm{X}_{0}$ & $\mathrm{X}_{0}$ & $\mathrm{X}_{0}$ & $\mathrm{X}_{0}$ & $\mathrm{X}_{0}$ & \\
\hline & $\mathrm{X}_{0}$ & $\mathrm{X}_{0}$ & $\mathrm{X}_{0}$ & $\mathrm{X}_{0}$ & $\mathrm{X}_{0}$ & $\mathrm{X}_{0}$ & $\mathrm{X}_{0}$ & \\
\hline & & $\mathrm{X}_{0}$ & $\mathrm{X}_{0}$ & $\mathrm{X}_{0}$ & $\mathrm{X}_{0}$ & $\mathrm{X}_{0}$ & & \\
\hline
\end{tabular}

Figure 3. Construction of hexagonal pixel 
Thus complete image can be constructed using spiral addressing scheme. Figure 4 gives a view of image constructed using spiral addressing technique using pixels in the power of 7 , as spiral addressing scheme assigns base 7 addresses to its hexagonal pixels.

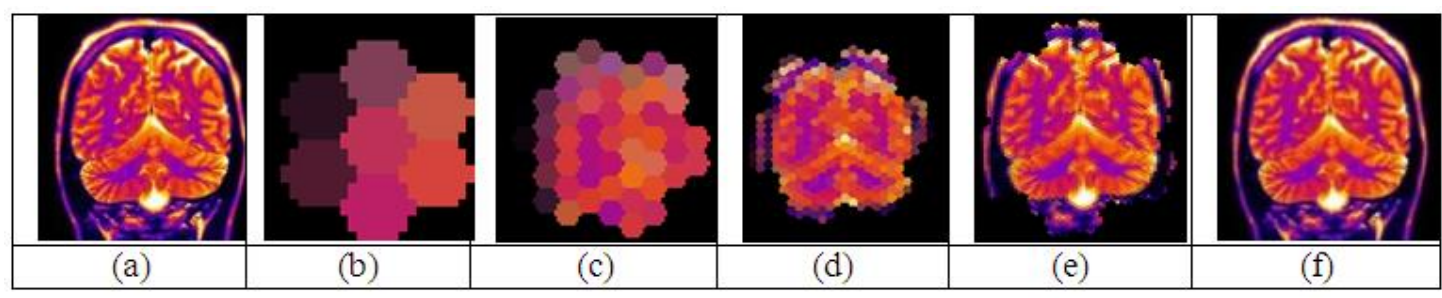

Figure 4. (a) Original square pixel image; conversion to hexagonal pixel image (b) using 7 pixels (c) using 72 pixels (d) using 73 pixels (e) using 74 pixels (f) using 75 pixels

\section{Step 2: Gabor filter enhancement}

Frequency and orientation representations of Gabor filters are similar to those of the human visual system, and they have been found to be particularly appropriate for texture representation and discrimination. In the spatial domain, the standard definition of a $2 \mathrm{D}$ Gabor filter is a Gaussian kernel function modulated by a sinusoidal plane wave [4]. It can be written as:

$$
h(x, y)=s(x, y) \cdot g(x, y)
$$

$\mathrm{s}(\mathrm{x}, \mathrm{y})$ is a complex sinusoid known as carrier; $\mathrm{g}(\mathrm{x}, \mathrm{y})$ is a 2-D Gaussian shape function known as envelope. The complex sinusoid is defined as follows:

$$
s(x, y)=e^{-j 2 \pi\left(u_{0} x+v_{0} y\right)}
$$

The 2-D Gaussian function is defined as follows:

$$
g(x, y)=\frac{1}{\sqrt{2 \pi} \sigma} e^{-\frac{1}{2}\left(\frac{x^{2}}{\sigma_{x}^{2}}+\frac{y^{2}}{\sigma_{y}^{2}}\right)}
$$

The 2D gabor filter can be written as

$$
h(x, y)=e^{-\frac{1}{2}\left(\frac{x^{2}}{\sigma_{x}^{2}}+\frac{y^{2}}{\sigma_{y}^{2}}\right)} \cdot e^{-j 2 \pi\left(u_{0} x+v_{0} y\right)}
$$

In this paper Gabor filter is designed using above stated equations for image enhancement and is applied on Hexagonal pixeled images; obtained from Step-1. Finally PSNR (peak signal to noise ratio) of an image of size $m \times n$ is calculated using the equation as follows:

$$
P S N R=10 \log _{10}\left(\frac{255^{2}}{M S E}\right)
$$

Where MSE is the mean square error and is calculated as

$$
M S E=\sum_{i=1}^{m-1} \sum_{j=1}^{n-1} \frac{\left(A_{i j}-B_{i j}\right)^{2}}{m \times n}
$$

\section{Results \& discussions}

In this paper a research has been made for improvement in PSNR of medical images using the above said approach. Figure 5 shows the results of pixel reconstruction and its 
enhancement using Gabor filter. Table 1 and figure 6 gives the comparison results of Gabor filter enhanced image at different values of sigma. The optimized results are underlined at $\sigma=$ $0.5 / \pi$ with highest PSNR.

\begin{tabular}{|c|c|c|c|}
\hline Image & $\begin{array}{l}\text { Original square pixel } \\
\text { Image }\end{array}$ & $\begin{array}{l}\text { Hexagonal pixel } \\
\text { image (pixel } \\
\text { reconstruction) }\end{array}$ & $\begin{array}{l}\text { Gabor filter } \\
\text { enhanced image } \\
(\sigma=0.5 / \pi)\end{array}$ \\
\hline \multicolumn{4}{|l|}{ MRI 1} \\
\hline \multirow{2}{*}{\multicolumn{4}{|c|}{ MRI 2}} \\
\hline & & & \\
\hline & (b) & (e) & (h) \\
\hline \multicolumn{4}{|l|}{ MRI 3} \\
\hline & (c) & (f) & (i) \\
\hline
\end{tabular}

Figure 5. (a, b, c) Square pixel images of MRI 1, MRI 2, MRI 3; (g, e, f) its conversion to hexagonal pixel (pixel reconstruction using spiral addressing technique; $(g, h, i)$ their enhancement using Gabor filter at $(\sigma=1 / \pi)$.

Table 1. Comparison of hexagonal pixeled enhanced images using Gabor filter for different values of $\sigma(\sigma=1,0.5 / \pi, 1 / \pi, 2 / \pi)$ in terms of PSNR.

\begin{tabular}{|c|c|c|c|c|}
\hline Image & $\begin{array}{c}\text { PSNR } \\
(\sigma=1)\end{array}$ & $\begin{array}{c}\text { PSNR } \\
(\sigma=0.5 / \pi)\end{array}$ & $\begin{array}{c}\text { PSNR } \\
(\sigma=1 / \pi)\end{array}$ & $\begin{array}{c}\text { PSNR } \\
(\sigma=2 / \pi)\end{array}$ \\
\hline MRI 1 & 77.9623 & $\mathbf{9 5 . 5 0 8 1}$ & 87.6234 & 80.0888 \\
\hline MRI 2 & 75.4007 & $\underline{\mathbf{7 9 . 3 3 4 8}}$ & 78.6669 & 76.9762 \\
\hline MRI 3 & 74.6020 & $\underline{\mathbf{1 2 2 . 5 5 3 3}}$ & 85.5955 & 77.0207 \\
\hline
\end{tabular}


Table 1 briefs the PSNR results obtained that have been calculated from Gabor filter enhanced hexaonal pixel image at different values of sigma. The results are calculated on three MRI images shown in Figure 5. Finally figure 6 shows a plot of PSNR value against sigma value and the results show that the highest Picture quality is obtained at $\sigma=0.5 / \pi$.

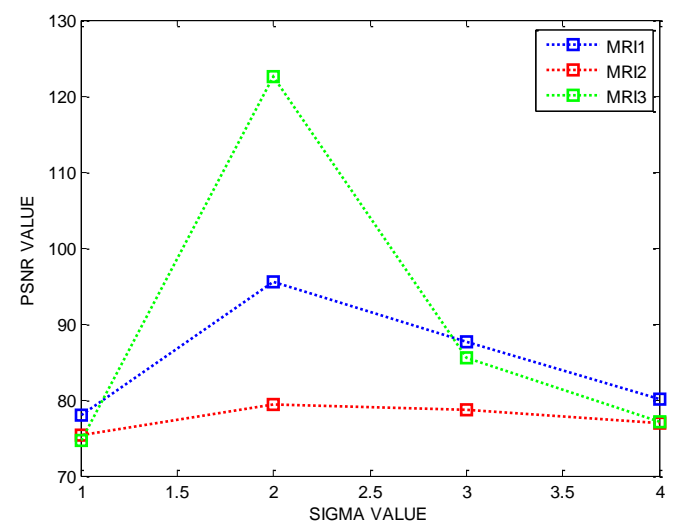

\section{Figure 6. Plot of sigma value versus PSNR value using Gabor filter on hexagonal pixelwed images at different values of sigma}

\section{Conclusion}

We proposed a work for enhancement of medical images using a noval pixel reconstruction and Gabor enhancement approach. The results of Gabor enhancement are evaluated by obtaining results at different values of sigma $(\sigma=1,0.5 / \pi, 1 / \pi, 2 / \pi)$. The optimum results are obtained at $\sigma=0.5 / \pi$. finally the experimental results show that the color enhancement using Gabor filter gives maximum improvement in the quality of an image in terms of PSNR, at a specific value of sigma.

\section{References:}

[1] M. Golay, "Hexagonal Parallel Pattern Transformation", IEEE Transactions on Computers, vol. 18, (1969), pp 733-740.

[2] R. M. Mersereau, "The Processing of Hexagonally Sampled Two- Dimensional Signals", Proceedings of the IEEE, vol. 67, (1979), pp 930-949.

[3] X. He, T. Hintz, Q. Wu, H. Wang and W. Jia, "A New Simulation of Spiral Architecture”, In Proc. IPCV, (2006), pp 570-575.

[4] S. Veni and K. A. Narayanankutty, "Gabor Functions for Interpolation on Hegagonal Lattice", International Journal of Electronics and Communication Technology, vol. 2, (2011), pp 15-19.

[5] S. Veni and K. A. Narayanankutty, "Vision-Based Hexagonal Image Processing using Hex-Gabor", SpringerVerlog Journal-Signal, Image and Video Processing, vol. 8, (2012), pp 317-326.

[6] B. Kumar, P. Gupta and K. Pahwa, "Square Pixel to Hexagonal Pixel Representation Techniques", International Journal of Signal Processing, Image Processing and Pattern Recognition, vol. 7, (2014), pp 137144.

[7] R. C. Gonzalez and R. E. Woods, "Digital image processing”, New Jersey: Prentice Hall, (2002).

[8] L. Middleton and J. Sivaswamy, "Hexagonal Image Processing - A Practical Approach", Springer-Verlag, London Limited, (2005). 


\section{Authors}

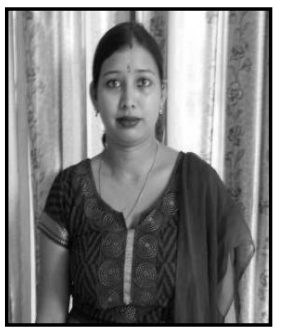

\section{Pooja Gupta}

Received her M. Tech from Punjab Technical University, Kapurthalla in 2009. She is currently working as Assistant Professor at Electronics and Communication Engineering Department at M. M. University Mullana. His research interests include Image Processing and Embedded System Design.

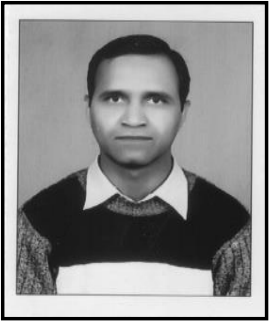

\section{Dr. Kuldip Pahwa}

Received his Ph.D from M. M. University, Mullana (Ambala) in 2013. $\mathrm{He}$ is currently working as Professor and Head at Electronics and Communication Engineering Department at M. M. University Mullana. His research interests include Antenna Design, Optical Communication and Wireless Communication. 